\title{
Use of 3-D Printers to Design, Build, and Test a Quadcopter Drone
}

\section{Dr. R. Radharamanan, Mercer University}

Dr. R. Radharamanan is currently working as Professor of Industrial Engineering and Director of Mercer Center for Innovation and Entrepreneurship (MCIE) at Mercer University in Macon, Georgia. He has forty two years of teaching, research, and consulting experiences. His previous administrative experiences include: President of International Society for Productivity Enhancement (ISPE), Acting Director of Industrial Engineering as well as Director of Advanced Manufacturing Center at Marquette University, and Research Director of CAM and Robotics Center at San Diego State University. His primary research and teaching interests are in the areas of manufacturing systems, additive manufacturing, rapid prototyping, robotics and automation, innovation and entrepreneurship, quality engineering, and product and process development. He has organized and chaired five international conferences, co-chaired two, and organized and chaired three regional conferences. He has received two teaching awards, several research and service awards in the United States and in Brazil. His present and past professional affiliations include ASEE, IIE, ASQ, SME, ASME, and ISPE. 


\title{
Use of 3D Printers to Design, Build, and Test a Quadcopter Drone
}

\begin{abstract}
The body,arms, and legsof a quadcopter drone were designed using two design softwares, 123D Design and MeshMixer. These parts were printed using two 3D printers: The MakerBot Replicator 2X was used to print the arms using ABS (Acrylonitrile butadiene styrene) material and the Flashforge Creator Pro printed the body and legs using PLA (Polylactic acid) material. The printed parts were tested for dimensional accuracy and surface roughness. The electronic parts for the drone consisted of one flight control, four electronic speed controllers, one transmitter, one receiver, four motors, four propellers, and one GPS. The 3D printed parts and the electronic components were assembled to make the prototype of the quadcopter drone. Through this hands-on project, the students were trained in two new and emerging manufacturing technologies: 3D printing and rapid prototyping as well as drone technology. Some of the difficulties encountered by the student team include assembly errors, sizing issues, and software incompatibility. Flight tests were performed and the errors identified and corrected. The results of the flying quadcopter drone designed, built, and tested are presented and discussed.
\end{abstract}

\section{Introduction}

This paper is one of the outcomes of the Mercer Summer Engineering Experience (MeSEE 2015), an Academic Training program, in which multidisciplinary student teams were trained in engineering labs and then worked on hand-on projects over a period of 10 weeks (30-40 hours/ week) in the lab environment, during 2015 Summer semester to complete the chosen projects. Three senior students (Ana Carolina Martins Rosa, Industrial Engineering; Victor Ferreira Bispo Santos, Mechanical Engineering; and Benedito Adilson Domiciano Neto, Mechatronics/ Automation Engineering) forming a multidisciplinary team worked on this project.

The overall objective of this project is to design, build, test, and fly a quadcopter drone within the ten weeks duration of the academic training. This includes:

1. Provide training to the student team in CAD softwares: use of 123D Design and MeshMixer to design parts;and 3D Printing and Rapid Prototyping: use of 3D printers (MakerBot Replicator $2 \mathrm{X}$ and Flashforge Creator Pro) to print the designed parts;

2. Review of literature and watching online tutorials related to 3D Printing and Designing and Building of a Drone (Do It Yourself) by the student team;

3. Design the quadcopter drone parts (arms, body, and legs) using 123D Design and MeshMixer;

4. Print the parts in 3D printers: 4 arms in the MakerBot Replicator $2 \mathrm{X}$ using ABS material; body (bottom and top part), and 4 legs in Flashforge Creator Pro using PLA material;

5. Measure the parts made of ABS and PLA for dimensional accuracy and surface roughness and compare the results; 
6. Purchase the electronic components/parts that are required to make the drone; Build the drone using the printed parts and the purchased electronic components;

7. Do the calibrations, conduct flying tests, correct the errors, and make the drone to fly;

8. Write the final reportwith the team members and prepare the poster for final presentation in consultation with the academic training advisor. Submit the final report and make the oral and poster presentation.

In addition, this project is designed to fully/partially satisfy some of the ABET's student learning outcomes that include:

b. An ability to design and conduct experiments, as well as to analyze and interpret data;

c. An ability to design a system, component, or process to meet desired needs within realistic constraints such as safety, manufacturability, and sustainability;

d. An ability to function on multidisciplinary teams;

g. An ability to communicate effectively (orally and written);

k. An ability to use the techniques, skills, and modern engineering tools necessary for engineering practice.

The 3D printers are capable of producing three-dimensional solid objects drawn in 3D software through an additive process, wherein the feedstock is applied layer by layer to form the threedimensional object ${ }^{1-3}$. The drones are instruments controlled from a distance by electronic and computational mechanisms. Many of them seem aero-modeling toys or even remote control helicopters, but the difference is in the technology employed, usually much more complex than mere toys. Its utilities go beyond conventional, they can be used either for leisure, commercial or military work.

Uniting these technologies, a drone was designed, built, and tested by the student team. All the parts of the drone were printed in the 3D printers: arms, body, and legs. The electronic parts were bought separately: motors, battery, charger, electronic speed controllers (ESCs), and remote control. All printed and purchased parts were put together and tested for the drone to fly.

Two softwares were used to design the parts of the drone: 123D Design and MeshMixer. These are compatible with the 3D Printers. Two 3D printersused to print the parts are: the MakerBot Replicator 2X and the Flashforge Creator Pro. Currently, both printers are available for training students in additive manufacturing/rapid prototyping at Mercer University School of Engineering. The MakerBot Replicator $2 \mathrm{X}$ was used to print the arms using ABS, a common thermoplastic polymer, very rigid and light, with a good balance of strength and flexibility. The Flashforge Creator Pro printed the body and legs using PLA, a biodegradable thermoplastic polyester, more efficient in certain types of molding than the ABS, because it tends to deform less after the application and releases less smoke upon reaching its melting point. The printed parts were measured for dimensional accuracy and the surface roughness measurements were made using a surface roughness tester. Also, flight test were performed and some errors were corrected for the drone to fly without errors. 


\section{Background Research}

\section{The 3D Printer}

The 3D printer is a machine allowing the creation of physical object from a three-dimensional digital model, typically by laying down many thin layers of a material in succession ${ }^{3}$. This is the main characteristic that distinguishes the 3D printers from other numerically controlled (CNC) machines where the production process is subtractive, meaning that the final object is achieved by removing the raw material using different mechanical tools ${ }^{4-5}$.

The 3D printer has become a good alley of Rapid Prototyping, because the process of this technology is easy to design, rapid to create, or replace ${ }^{6-7}$. Manufacturers and product developers used to find prototyping a complex, tedious, and expensive process that often impeded the developmental and creative phases during the introduction of a new product and with this new term and the $3 \mathrm{D}$ printer, all this process has become easy to manage and fast to accomplish ${ }^{7}$.

\section{The Drone}

The drones were designed for military purposes. Inspired by the German flying bombs, the V-1 type, and those harmless radio-controlled airplane models, these flying machines of the latest generation are designed, engineered and built to be used in very dangerous missions that cannot be performed by humans in the areas of military intelligence, support and control of artillery fire, air support for infantry and cavalry troops on the battlefield, cruise missiles control, urban patrolling, coastal, environmental activities and borders, search and rescue activities, among others $^{8}$.

\section{The Electronic Parts of the Drone}

Every drone has the basic parts that are required for it to fly and each part has its functionality and usefulness. Starting with the ESC (Electronic Speed Controller), it does two important things for the drone. First it converts the battery voltage down to $5 \mathrm{~V}$, using which the receiver runs. Second, it converts the DC power from the battery to an AC current, which is required by the motor $^{9}$.The Turnigy $9 \mathrm{X}$ is a radio channel dedicated to $2.4 \mathrm{GHz} 8$-channels and is manufactured by Flysky as the FS-TH9X. This has the remote control and the transmitter. When programming both, one can send by the transmitter the correct information to the remote control and see it flying.

The DJI Naza-M V2 is a powerful flight controller for enthusiast, commercial and industrial flyers. It is easy to install, simple to configure and above all, extremely stable. The Naza-M V2 boasts have the extraordinary stability one would expect of all DJI flight control systems and combines it with unparalleled maneuverability with and without GPS. Built into it are automatic GPS course correction plus GPS and compass interference monitoring, which combine to offer more stable flight and minimal magnetic interference. If the connection between the multirotor and the remote control are disconnected during the flight, a failsafe system will activate. Provided there was enough GPS signal at the time of the disconnection, the multirotor will fly back to its point of takeoff and land automatically ${ }^{10}$. 


\section{Methodology}

\section{Design of the Drone}

The 123D Design software helped to give the shape to the parts: arms, body, and legs; then MeshMixer helped to fix the corner from the part using the tool, make it solid, that gave the final look to the part. Figure 1 shows the CAD of the arm in the MeshMixer ready to print.

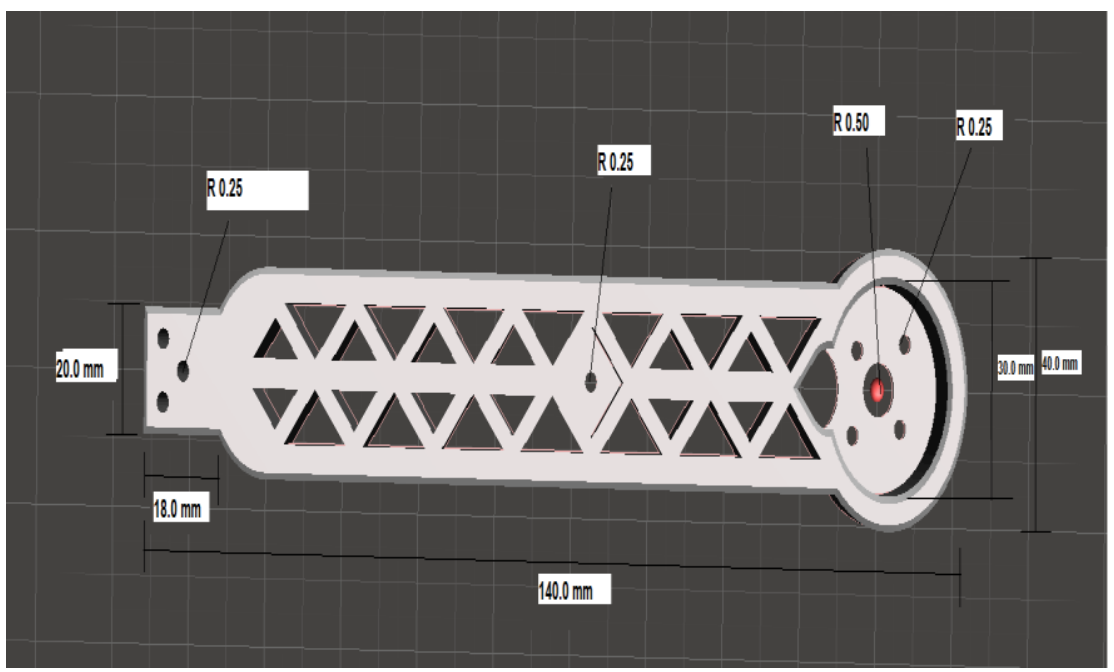

Figure 1: CAD of the arm

The four arms were printed in the MakerBot Replicator $2 \mathrm{X}^{11} 3 \mathrm{D}$ printer using ABS material. The ABS has a good balance of strength and flexibility besides being very rigid and light, it was a better choice for the arms than the PLA. The temperature used to print the arms was $230^{\circ} \mathrm{C}$; the platform temperature was $110^{\circ} \mathrm{C}$; the layer height was $0.20 \mathrm{~mm}$; and the infill was $75 \%$. Figures 2(a) and 2(b) show details of dimensions and print setting respectively for the arm.

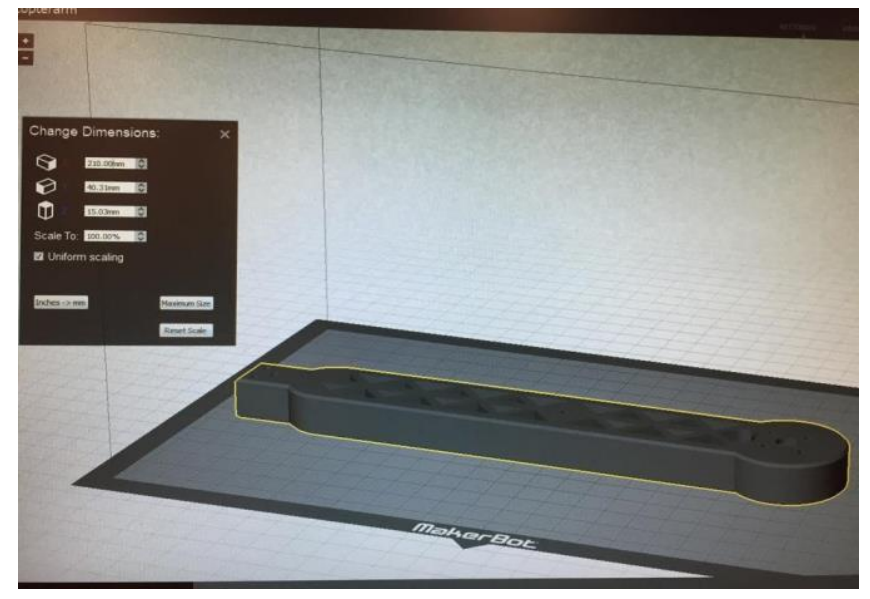

(a) Dimensions used to print the arm

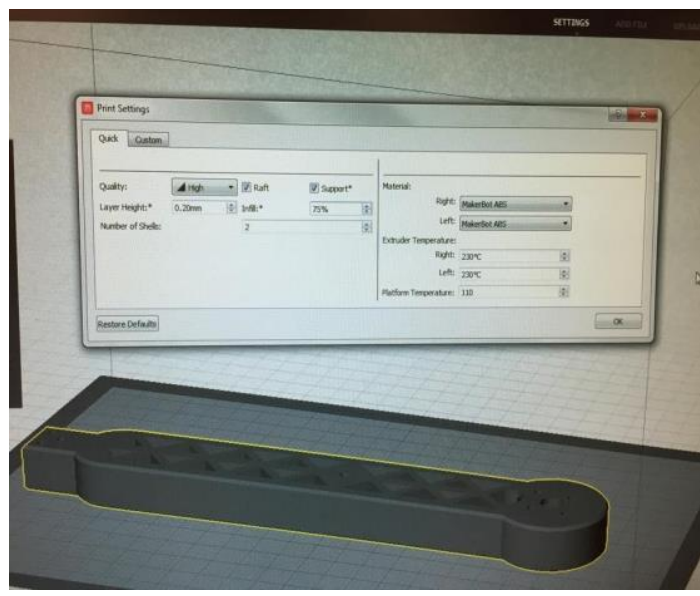

(b) Print setting of the arm Figure 2: Details of dimensions and print setting for the arm

The body was divided in two parts, the bottom part and the top part, when put together it becomes a box. It was printed in the Flashforge Creator Pro ${ }^{12}$ 3D Printer using PLA material. 
The PLA is more efficient in molding than the ABS; also, because it has a lower melting point, it results in more resistant objects at the end. Furthermore, because the PLA is less viscous when in liquid state, it requires less force of the extruder to expel the material, which can ensure a little more durability for the equipment. Thus, the body in which the electronic parts are connected and assembled to form the brain of thedrone, must be protected from drops, and PLA can ensure better protection than $\mathrm{ABS}$. The temperature used to print the body was $220^{\circ} \mathrm{C}$; the platform temperature was $110^{\circ} \mathrm{C}$; the layer height was $0.20 \mathrm{~mm}$; and the infill was $75 \%$. Figures 3 and 4 show the CAD drawings of the bottom/top of the box and leg respectively, both were printed in PLA material in the Flashforge Creator Pro.

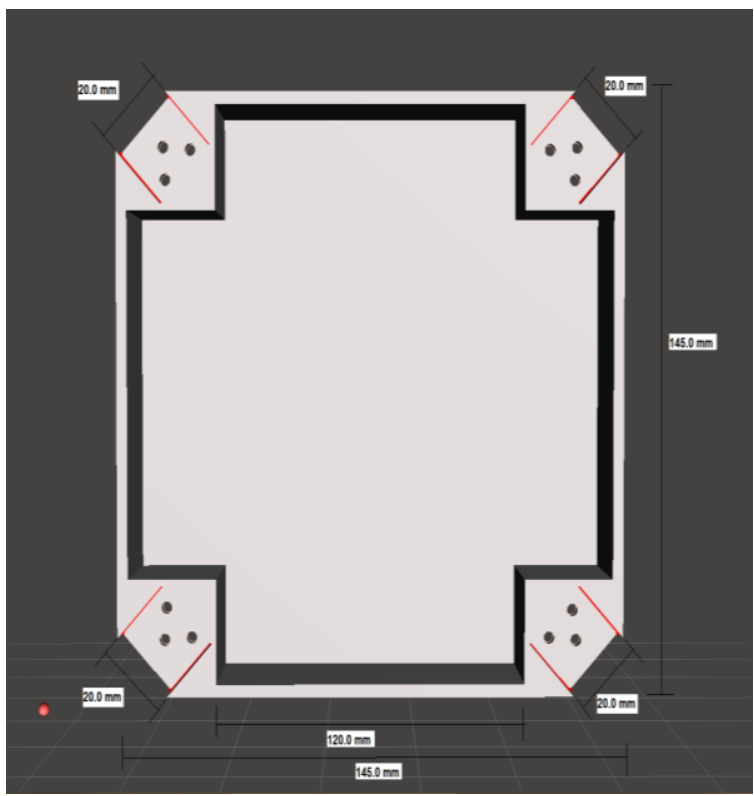

(a) Bottom box

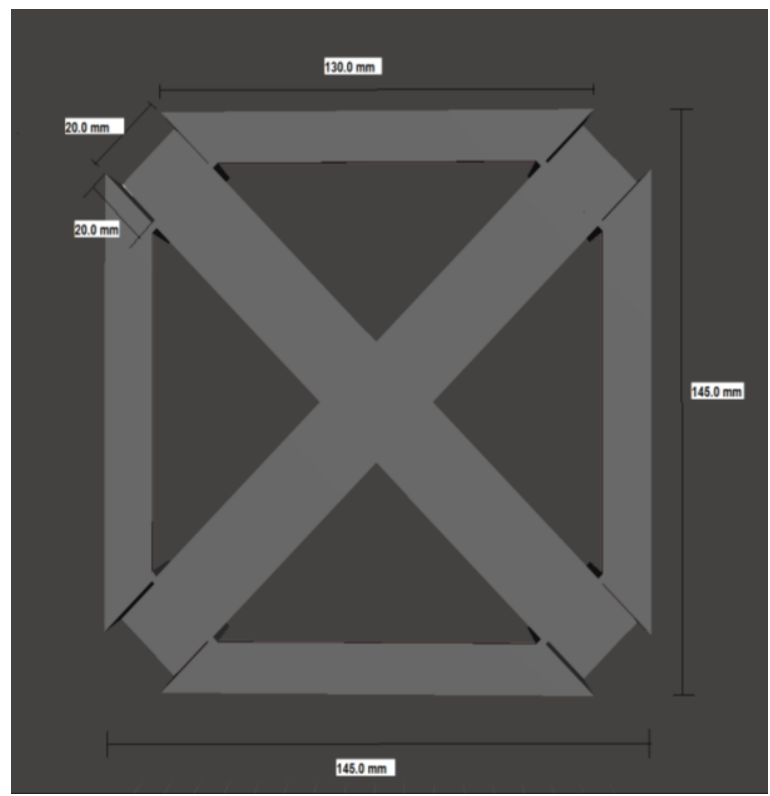

(b) Top box

Figure 3: CAD of bottom and top of the box

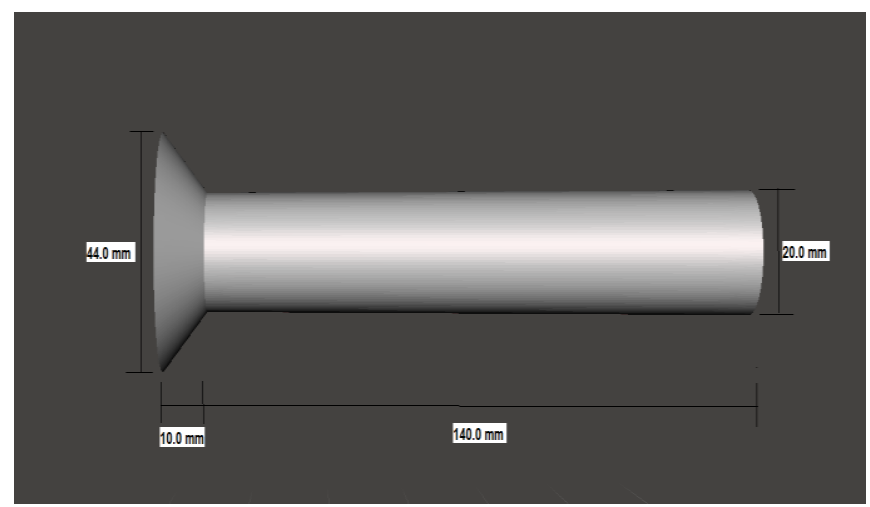

Figure 4: CAD of the leg

Figures 5(a) and 5(b) show the print settings and dimensions respectively of the bottom box in the MakerBot software. 


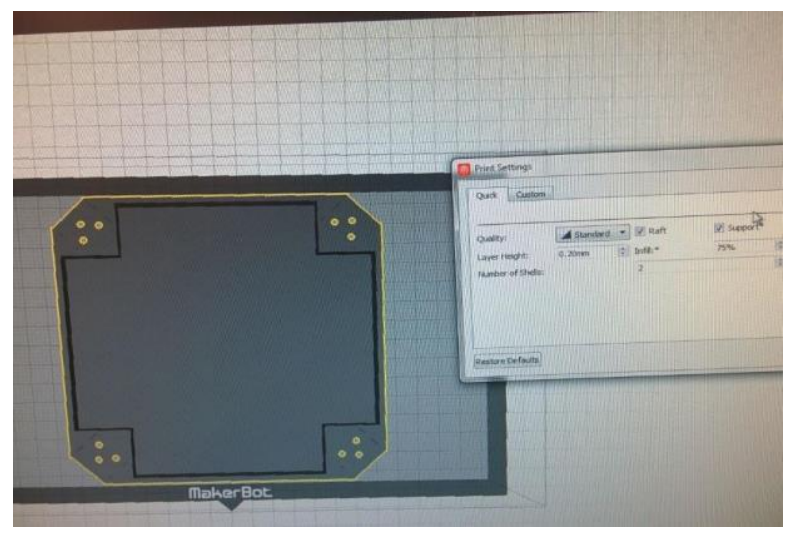

(a) Print setting of the bottom box

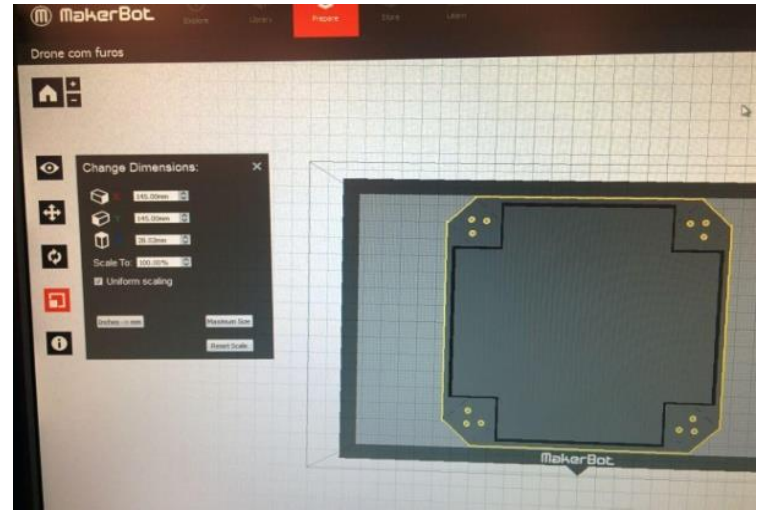

(b) Dimensions of the bottom box

Figure 5: Print settings and dimensions of the bottom box in the MakerBot software

\section{Materials for the Electronic Assembly of the Drone}

To truly have a drone and see it flying the following list of materials is needed:

- 4 motors - NTM Prop Drive Series 28-26A 1200kv / 250w

- Propellers - 10x4.5 SF Props 2pc Standard Rotation/2 pc RH Rotation (Black)

- 4 ESC - TURNIGY Plush 30amp Speed Controller

- Remote control and transmitter -Turnigy 9X 9Ch Transmitter w/ Module \& 8ch Receiver (Mode 2) (V2 Firmware)

- Battery - Turnigynano-tech 3000mah 3S 25 50C Lipo Pack

- Charger for the battery - Turnigy Accucel-8 150W 7A Balancer/Charger

- Flight Controller - DJI Naza-M V2

- Wires - 20AWG Soft Flexible Silicone Wire - Black + Red and 16AWG Soft Flexible Silicone Wire - Black + Red

- Connectors - XT60

- Screw - M3x30x0.5

- Zip ties

- Sockets - 3 x 30mm Socket Head Cap Screw 10pcs Class 12.9 Hard Alloy Steel

- CA Glue

- Banana Plug

\section{Building the Drone}

\section{Mounting the Motors}

With the parts printed, it is possible to start the construction of the drone. Each arm requires a motor and an ESC. First, each motor was fit to the location designed for it and fixed with four screws.

\section{Mounting the Electronic Parts}

Now it is time to wire up the transmitter, the flight controller, and the ESCs. The DJI Naza-M V2 is the flight controller, and in its box there is the main controller, the servo cables, LED module, PMU (Power Management Unit) module, the GPS, and compass module. First, the PMU module 
was soldered to the battery, the red wire to the positive pole and the black one to the negative pole of the battery. After soldering was completed, the main controller was mounted. The arms 1 and 2 were chosen to be in the nose direction of the aircraft, so the arrow on the main controller must be the same as the nose direction, here the locations were defined as M1, M2, M3, and M4, the M1 matches with arm 1, M2 matches with arm 2, and so on. Each ESC has a 3PIN signal line to be connected into the main controller, so it was connected from M1 through M4 port on the main controller one by one. Then the 3PIN port of the PMU was connected to the X3 channel on the main controller, the 4PIN port on the PMU to the EXP channel of the main controller, and the LED module to the main controller. After, the transmitter was connected to the Naza using a servo cable. The S-Bus receiver in the transmitter was connected to the $\mathrm{X} 2$ channel of the Naza.At the end, the GPS module was plugged into the GPS port on the PMU. Figure 6 shows all electronic parts connected and fitted in the bottom box.

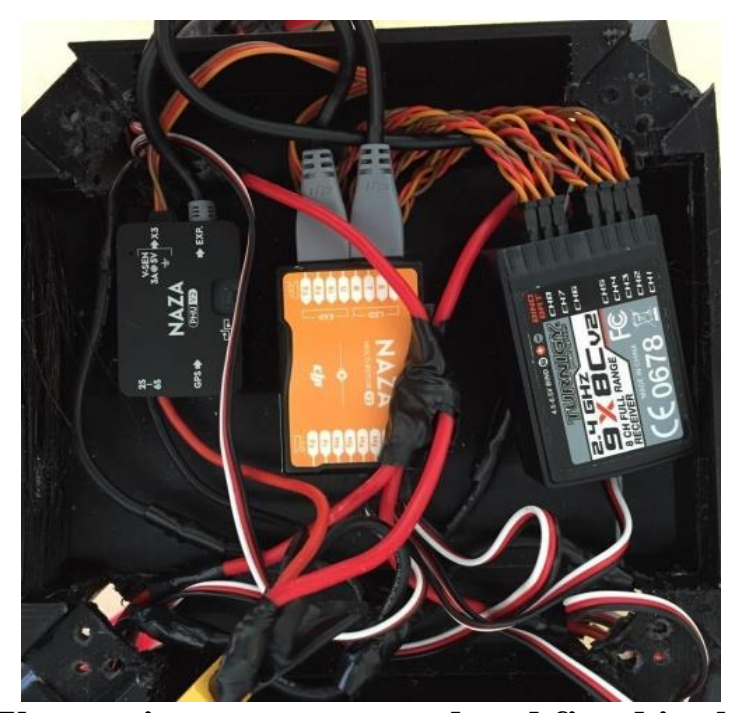

Figure 6: Electronic parts connected and fitted in the bottom box

\section{Configuration}

\section{Electronic Speed Controller (ESC) Calibration}

Electronic speed controllers are responsible for spinning the motors at the speed requested by the autopilot. Figure 7 shows a Turnigy-Electronic Speed Controller used. Most ESCs need to be calibrated so that they know the minimum and maximum PWM (Pulse Width Modulation) values that the flight controller will send. Before calibrating ESCs, make sure that the copter has no propellers on it and that the APM (ArduPilot) is not connected to a computer via USB and the Lipo battery is disconnected.

The four ESCs were calibrated following these simple steps:

1. Connect the ESC to channel 3, which is responsible for the throttle channel, in the receiver.

2. Turn on the transmitter, move the stick throttle to the top, and turn on the ESC.

3. Wait for the ESC emit the musical tone, the regular number of tones indicating the battery's cell count, in this case 3 beeps $=$ LIPO 3S. Then an additional two beeps to indicate that the maximum throttle has been captured. 
4. Pull the transmitter's throttle stick to its minimum position.

5. The ESC should emit a long beep indicating that the minimum throttle has been captured and the calibration has been completed ${ }^{13}$.

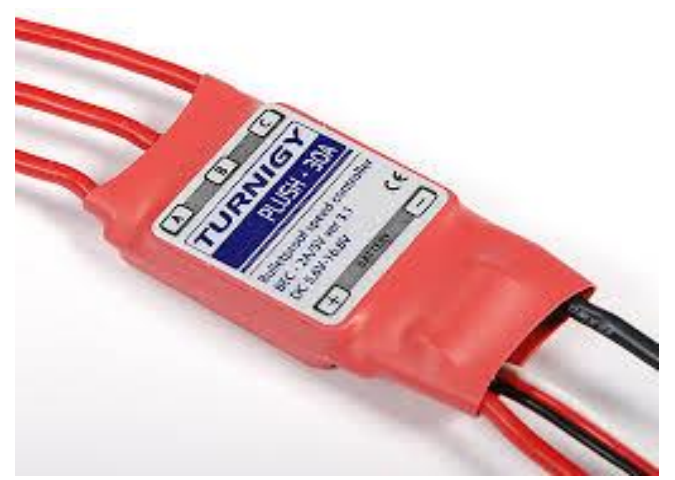

Figure 7: Turnigy ESC 30A

\section{Flight Controller CalibrationNAZA-M V2}

The NAZA-M V2 is a powerful flight controller that is composed for the MC (Main Controller), PMU (Power Management Unit), LED, and GPS/Compass. The controller Turnigy RF 9X V2 and the DJI NAZA-M V2 flight controller were configured using the DJI NAZA Assistantsoftware ${ }^{10}$.

\section{Naza-M-V2 Compass Calibration}

Calibration for the GPS module - Calibration Procedure:

1. Switch on the transmitter, and then power on autopilot system ${ }^{14}$.

2. Quickly switch the control mode switch from Manual Mode to GPS ATTI Mode and back to Manual Mode for 6 to 10 times, The LED indicator will turn on constantly yellow.

3. Hold themultirotor horizontal and rotate it around the gravitational force line (about $360^{\circ}$ ) until the LED changes to constant green, and then go to the next step.

4. Hold themultirotor vertically and rotate it (its nose downward) around the gravitational force line (about $360^{\circ}$ ) until the LED turns off, meaning the calibration is completed.

5. If the calibration was successful, calibration mode will exit automatically. If the LED keeps flashing quickly Red, the calibration has failed. Switch the control mode switch one time to cancel the calibration, and then restart from step 2.

\section{Turnigy 9X Transmitter Configuration}

The transmitter was configured in type Acro and mode 2, as shown in Figures 8 and 9, which are the best settings for a quadcopter drone. When the Turnigy 9x transmitter is in mode 2, the left stick controls the throttle and the rudder, and the right stick controls the ailerons and the elevator ${ }^{15}$. 


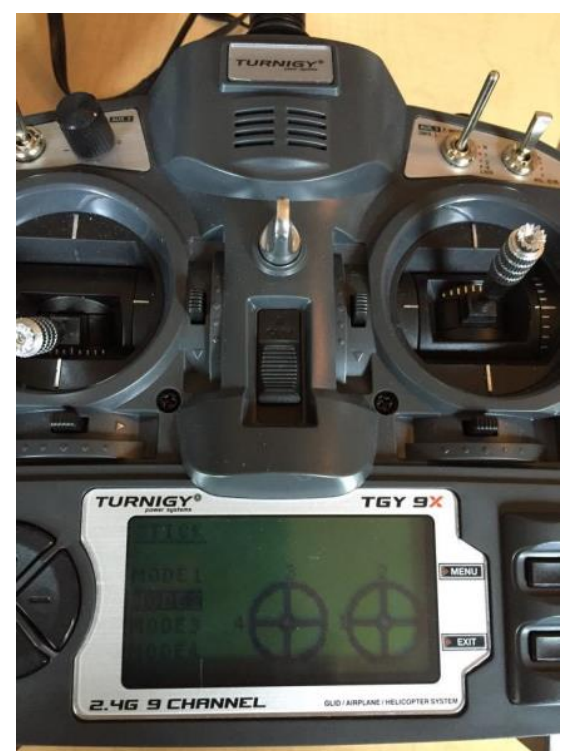

Figures 8: Turnigy transmitter configuration

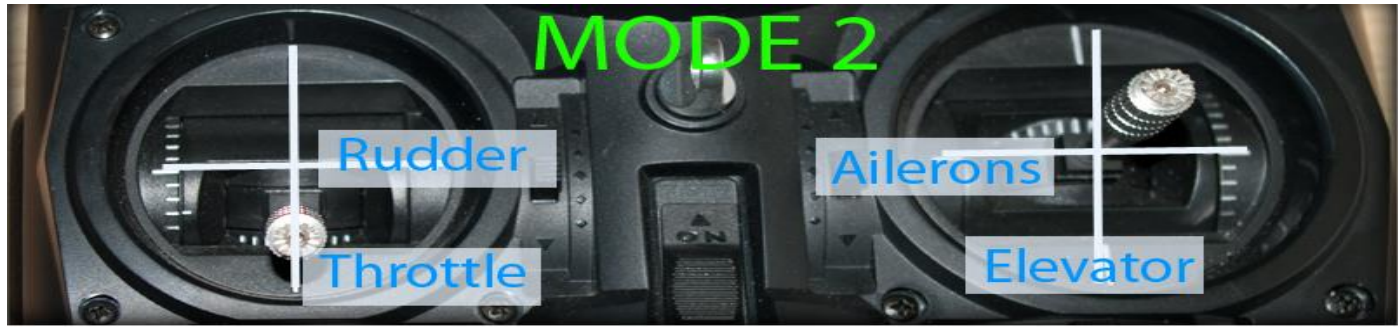

Figure 9: Mode 2 configuration

\section{Mounting the propellers}

An easy way to find out the direction of the propeller is looking at it visually. When spinning clockwise, the leading edge should always be in the front. But the easiest way to tell if a prop is counterclockwise is by looking at the letters and numbers on it.

\section{Results and Discussions}

The dimensions of the 3D printed parts, body, arms, and legs were measured using dial gages and found to be within the tolerance limits. A surface roughness tester, SRT- $620^{10}$ was used to measure the surface quality of the printed parts (Figure 10). This test presented the surface quality of the material printed in numerical form, making it easier to analyze.

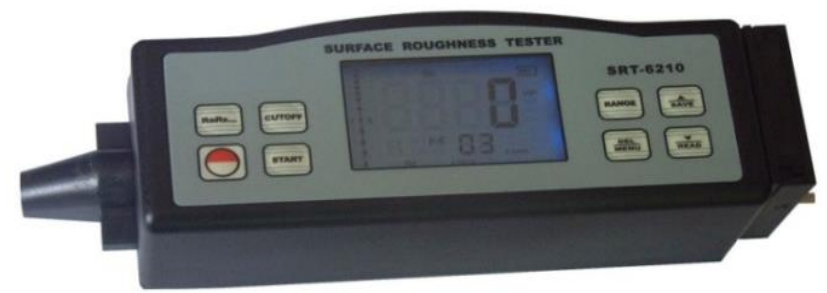

Figure 10: Surface roughness tester, SRT-6210 


\section{Surface Roughness Test}

Surface roughness is a component of surface texture. It is measured by the deviations in the direction of the normal vector of a real surface based on its ideal form ${ }^{16}$. Large deviations show that the surface is rough and small deviations present a smooth surface ${ }^{17}$. The surface roughness parameters are measured using the equations presented in Table 1.

Table 1: Surface roughness parameters

\begin{tabular}{|c|c|c|}
\hline \multirow[t]{2}{*}{$\mathrm{R}_{\mathrm{a}}$} & \multicolumn{2}{|c|}{$R_{a}=\frac{1}{n} \sum_{i=1}^{n}\left|Y_{i}\right|$} \\
\hline & \multicolumn{2}{|c|}{ Arithmetic Average } \\
\hline \multirow[t]{2}{*}{$\mathrm{R}_{\mathrm{z}}$} & $R_{z}=\frac{\sum_{i=1}^{5} Y_{i}+\sum_{i=1}^{5} Y_{v}}{5}$ & $\begin{array}{l}\text { Where: } \\
\text { Yi = Maximum Height } \\
\text { Yv = Minimum Valley }\end{array}$ \\
\hline & \multicolumn{2}{|c|}{$\begin{array}{l}\text { Average Height of } 5 \text { Highest Peaks } \\
\text { and } 5 \text { Lowest Valleys }\end{array}$} \\
\hline \multirow{2}{*}{$\mathrm{R}_{\mathrm{t}}$} & & \\
\hline & \multicolumn{2}{|c|}{ Maximum Height of the Profile } \\
\hline \multirow{3}{*}{$\mathrm{R}_{\mathrm{q}}$} & \multirow{2}{*}{\multicolumn{2}{|c|}{$R_{q}=\frac{\sum_{i=1}^{n} Y_{i}^{2}}{n}$}} \\
\hline & & \\
\hline & \multicolumn{2}{|c|}{ RMS (Root Means Square) Value } \\
\hline
\end{tabular}

\section{Results of Roughness Measurements}

Tables 2 and 3 present the roughness measurements $\left(R_{a}, R_{z}, R_{t}\right.$, and $\left.R_{q}\right)$ made on printed parts using ABS and PLA materials. From the results, it can be concluded that the two materials show a very similar quality, with slight advantage for the PLA material. Also, it can be noted that the average roughness using PLA is more regular, while with the ABS shows a greater variation between prints ${ }^{18}$.

Table 2: ABS parts - Roughness measurements in $\mu \mathrm{m}$

\begin{tabular}{|c|l|c|c|c|c|c|}
\hline \multicolumn{7}{|c|}{ ABS Material } \\
\hline \multicolumn{2}{|c|}{} & Arm 1 & Arm 2 & Arm 3 & Arm 4 & Average \\
\hline $\mathrm{R}_{\mathrm{a}}$ & Mean & 2.740 & 4.781 & 5.331 & 2.343 & 3.798 \\
\hline & Standard Deviation & 1.065 & 1.557 & 0.779 & 0.792 & 1.048 \\
\hline $\mathrm{R}_{\mathrm{z}}$ & Mean & 7.748 & 13.518 & 15.073 & 6.625 & 10.741 \\
\hline & Standard Deviation & 3.013 & 4.402 & 2.202 & 2.241 & 2.965 \\
\hline $\mathrm{R}_{\mathrm{q}}$ & Mean & 3.357 & 6.751 & 7.281 & 3.306 & 5.228 \\
\hline & Standard Deviation & 1.359 & 2.657 & 1.157 & 1.239 & 1.603 \\
\hline $\mathrm{R}_{\mathrm{t}}$ & Mean & 7.825 & 13.651 & 15.223 & 6.691 & 10.848 \\
\hline & Standard Deviation & 3.042 & 4.446 & 2.225 & 2.264 & 2.994 \\
\hline
\end{tabular}


Table 3: PLA parts - Roughness measurements in $\mu \mathrm{m}$

\begin{tabular}{|c|l|c|c|c|}
\hline \multicolumn{4}{|l|}{ PLA Material } & \\
\hline \multicolumn{2}{|l|}{} & Upper Box & Lower Box & Average \\
\hline $\mathrm{R}_{\mathrm{a}}$ & Mean & 3.844 & 3.656 & 3.750 \\
\hline & Standard Deviation & 1.400 & 0.892 & 1.146 \\
\hline $\mathrm{R}_{\mathrm{z}}$ & Mean & 10.869 & 10.339 & 10.604 \\
\hline & Standard Deviation & 3.957 & 2.522 & 3.240 \\
\hline $\mathrm{R}_{\mathrm{q}}$ & Mean & 4.944 & 4.824 & 4.884 \\
\hline & Standard Deviation & 1.555 & 1.111 & 1.333 \\
\hline $\mathrm{R}_{\mathrm{t}}$ & Mean & 10.978 & 10.440 & 10.709 \\
\hline & Standard Deviation & 3.997 & 2.547 & 3.272 \\
\hline
\end{tabular}

\section{Flight Tests}

Test 1 - In the first flight test, there were some problems, including the failure of one of the propellers - Figure 11(a). The propeller was glued and some of the control settings revised, including changing the flight mode to one in which the GPS is not used. The test was not successful.

Test 2 - Again, some problems occurred with the drone, two of the propellers went higher than others, causing it to turn and, once again, breaking some propellers - Figure 11(b). A new kit of propellers was purchased for replacement, and finally the problem was found, the configuration of the propellers was wrong, the engines were reversed. The test was not successful.

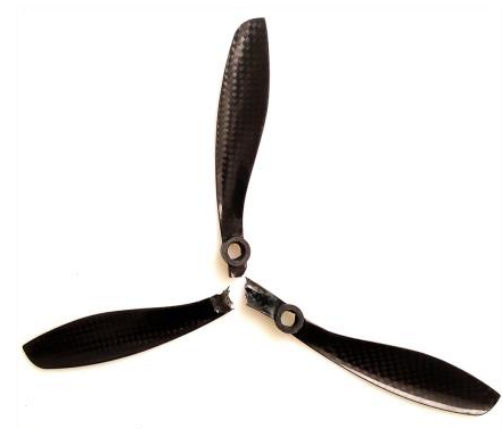

(a)

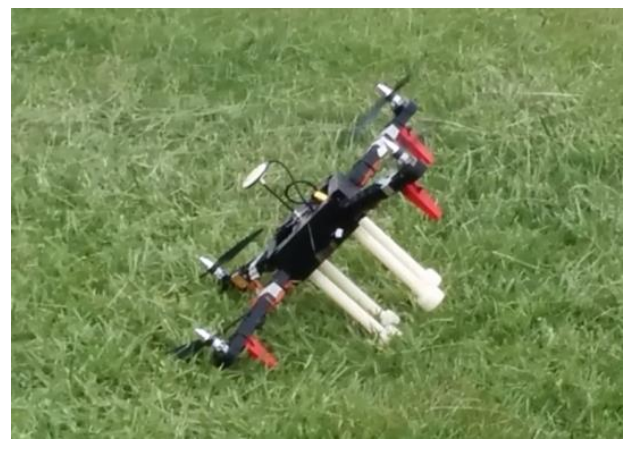

(b)

Figure 11: (a) Test 1 - One broken propeller; (b) Test 2 - Three broken propellers

Test 3 - With new propellers and engines in the right order, the third test finally worked. The drone could take off, stay stable, and reach the expected altitude. The test was successful this time.

Figure 12 shows the completed ready to fly drone. The link for the video of the flying drone is: https://youtu.be/JwQzwoAvHg8. 


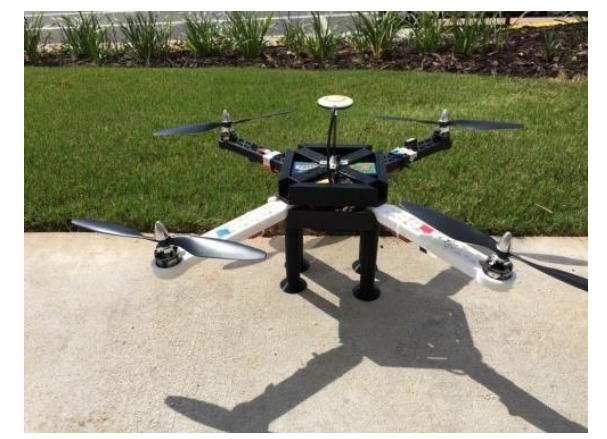

Figure 12: Completed ready to fly drone ${ }^{18}$

\section{Student Learning Outcomes and Asssessment}

The following ABET's student learning outcomes were fully/partially achieved by the student team through this project:

b. An ability to design and conduct experiments, as well as to analyze and interpret data: Drone parts were designed and 3D printed; measurements were made on 3D printed parts and the results were analyzed, compared, and interpreted; and flight tests were conducted;

c. An ability to design a system, component, or process to meet desired needs within realistic constraints such as safety, manufacturability, and sustainability: Drone parts were designed, 3D printed, assembled, tested, and the drone is safe to fly;

d. An ability to function on multidisciplinary teams: The student team was multidisciplinary (industrial, mechanical, and mechatronics) and they were able to work together in the lab environment and also achieve the overall objective of this project within the 10 weeks period;

g. An ability to communicate effectively (orally and written): The student team submitted the written report and the poster; They made the oral and poster presentation at the end of the academic training period;

k. An ability to use the techniques, skills, and modern engineering tools necessary for engineering practice: The student team used the techniques, skills, and engineering tools they learnt and practiced to successfuly complete this hands-on project within the academic training period of 10 weeks.

Assessment made on this project by student peers and the faculty members indicated that the student team fulfilled their initial goals and objectives and this project was rated as one of the best projects done by the student teams under Mercer Summer Engineering Experience (MeSEE 2015) Academic Training program with an average score greater than 4 in the 5-level Likert scale in all five ABET's student learning outcomes (b, c, d, g, and k) considered for this project.

\section{Conclusions and Recommendations}

The studentswere trained in using 123D Design and MeshMixer and two emerging technologies: 3D printing and rapid prototyping as well as drone technology. The students learnt to use 3D printers and print parts using two different materials (ABS and PLA). They were able to measure the printed parts for dimensional accuracy and surface roughness and compare the results. They were able to build, calibrate, and make the drone to fly. The overall objective of this project was to design, build, test, and fly a drone and it was successfully achieved by the student team. 
Assessment made by student peers and the faculty members indicated that the student team on average scored greater than 4 in the 5-level Likert scale in all five ABET's student learning outcomes (b, c, d, g, and k) considered for this project.

\section{Challenges and Lessons Learned}

Several problems arose during the process, like delay in the arrival of some materials, problems with the 3D printer, assembly errors, sizing issues, software incompatibility, among others. Some parts were replaced such as propellers, for example, due to a fall that broke them during a flight test. Excluding unforeseen problems mentioned above, there were the challenges of understanding the operation of each part and each component. These problems were solved referring to resources such as internet, reference manuals, and books as well as watching online tutorials and reading related articles. Focus and dedication of the student team was the key to successfully complete the project and make the drone flying.

\section{Recommendations}

As recommendation for anyone who wants to make their own drone the following suggestions are made: Take proper training in CAD and use of 3D printers; conduct background research on rapid prototyping and drone technology; read carefully the related materials (reference manuals, books, articles, internet resources, etc.) and watch online tutorials; never do anything when you are not sure; and take proper safety measures while conducting the flying tests.

\section{Acknowledgements}

This project was funded by the Mercer Summer Engineering Experience (MeSEE) program of Mercer University School of Engineering during the Summer semester of 2015.

\section{References}

[1] "3D Printing Basics: The Free Beginner's Guide - 3D Printing Industry", May 2014. 3dPrinting Industry.com/3d-printing-basics-free-begineers-guide/, Retrieved July 14, 2015.

[2] M. Petronzio, "How 3D Printing Actually Works", Mashable, March 28, 2013, meshable.com/2013/03/28/3dprinting explained/, RetrievedJuly 14, 2015.

[3] B. Evans, "Practical 3D Printers: The Science and Art of 3D Printing", A Press, 2012.

[4] I. Gibson, D. Rosen, and B. Stucker, Additive Manufacturing Technologies: Rapid Prototyping to Direct Digital Manufacturing, Springer Verlag, 2010.

[5] A. Gebhardt, "Understanding Additive Manufacturing: Rapid Prototyping, Rapid Tooling, Rapid Manufacturing, Hanser Publishers, 2012.

[6] C. K. Chua, K. F. Leong, and C. S. Lim, "Rapid Prototyping: Principles and Applications", World Scientific, Third Edition, 2010. 
[7] D. Bryden, “CAD and Rapid Prototyping for Product Design”, Laurence King Publishing, 2014.

[8] "History of the Drone." Doctor Drone. N.p., February 22, 2015. Retrieved July 14, 2015.

[9] "ESC (Electronic Speed Controller)",www.Instructables.com/FoamboardRC, Retrieved July 14, 2015.

[10] "Naza-M V2 - Features | DJI." CreateDJI. DJI, n.d. Retrieved July 14, 2015.

[11] "MakerBot Replicator 2X User Manual", www.makerbot.com/Replicator2X,Retrieved July 14, 2015.

[12] "Flashforge Creator Pro 3D Printer User Manual", www.flashforge-usa.com/creator-pro/, Retrieved July 14, 2015.

[13] "Electronic Speed Controller (ESC) Calibration", APM Copter, 3DRobotics, n.d., Retrieved July 14, 2015.

[14] "DJI Autopilot System Control Modes", DJI Wiki. N.p., 21 Jan. 2013. Retrieved July 14, 2015.

[15] "Configuring Turnigy 9x with arducopter - DIY Drones", diydrones.com/profiles/blogs/configuring-turnigy9x-with-arducopter, January 4, 2013, Retrieved July 14, 2015.

[16] "Roughness Tester Features 1. SRT-6210". Bikesu. BIKESU Technology Co., 2014. Retrieved July 14, 2015.

[17] "Surface Roughness - Formulas". Wikipedia, Wikimedia Foundation, 17 May 2015. Retrieved July 14, 2015.

[18] A. C. M. Rosa, B. D. Neto, and V. F. B. Santos, "Use of 3D Printer to Design, Build, and Test a Drone", Report, MeSEE 2015, pp. 1-54. 